\title{
PROTOCOLOS DE CARGA PARA PUENTES DE ACERO SUJETOS A BAJOS FACTORES DE SOBRERRESISTENICA
}

\section{TESTING'S LOADING PROTOCOLS IN STEEL BRIDGES SUBJECTED TO LOW OVERSTRENGTH FACTOR}

\author{
Jessica Fernanda Sarango Rodríguez ${ }^{1}$, Víctor Alejandro Calderón González ${ }^{2}$ \\ (1) Estudiante de Ingeniería Civil, Departamento de Ciencias de la Tierra y la \\ Construcción, Universidad de las fuerzas Armadas-ESPE, Sangolquí Ecuador, \\ ifsarango@espe.edu.ec
}

(2) Ph.D. student in Civil Engineering, Department of Civil, Construction, and Environmental Engineering, North Carolina State University, Raleigh United States, vacalder@ncsu.edu

Recibido: Enero, 2019 Aceptado: Abril, 2019

\section{RESUMEN}

Como parte del Proyecto Anchorage Port Access Bridge, se llevó a cabo un estudio acerca de los diferentes protocolos de carga aplicados en distintas secciones de vigas principales en marcos de puentes de acero. Se aplicarán distintos ciclos dinámicos de carga con el fin de capturar el comportamiento particular del puente. Este se caracteriza por la cedencia de las vigas principales antes que las columnas. Para ello se lleva a cabo un análisis computacional en SeismoStruct, que comprende un análisis pushover-estático y uno de historia en el tiempo, a fin de determinar en qué ciclo la columna y la viga entran en cedencia, luego se compara los resultados entre protocolos para determinar qué historial de carga permite analizar la respuesta de cada elemento.

Palabras claves: bajo factor de sobre resistencia, protocolo de carga cargas símicas, cargas dinámicas, ensayo cuasi- estático, conexión viga-columna, cedencia de la viga principal, puentes de acero.

\section{ABSTRACT}

As part of the Anchorage Port Access Bridge Project, a study of different loading protocols applied to diverse sections of cap beams in steel bridge frames was carried out. Different dynamic loading cycles will be applied in order to capture the particular behavior of the bridge. This is characterized by the yield of the cap beams before the columns. To do this, a computational analysis is developed in SeismoStruct, which includes a static-pushover analysis and a time history analysis in order to determine in which cycle the column and the beam enter in sequence, 
then the results are compared between protocols to determine which load history allows to analyze the response of each element.

Keywords: low overstrength factor, loading protocol, seismic loads, dynamic loads, quasi-static test, beam-column connection, cap beam yielding, steel bridges.

\section{INTRODUCCIÓN}

El puente Anchorage Port Access (APA) fue construido en 1972 con normas sísmicas diferentes a las actuales. Estas normas han evolucionado mucho sobre todo para regiones como Alaska que tienen alta peligrosidad sísmica (Alaska Seismic Hazards Safety Commission (ASHSC), 2019). Por lo tanto, es importante establecer si el puente cumple con la filosofía de diseño sísmico actual. En las Figuras 1 y 2 se muestran vistas en elevación y planta.

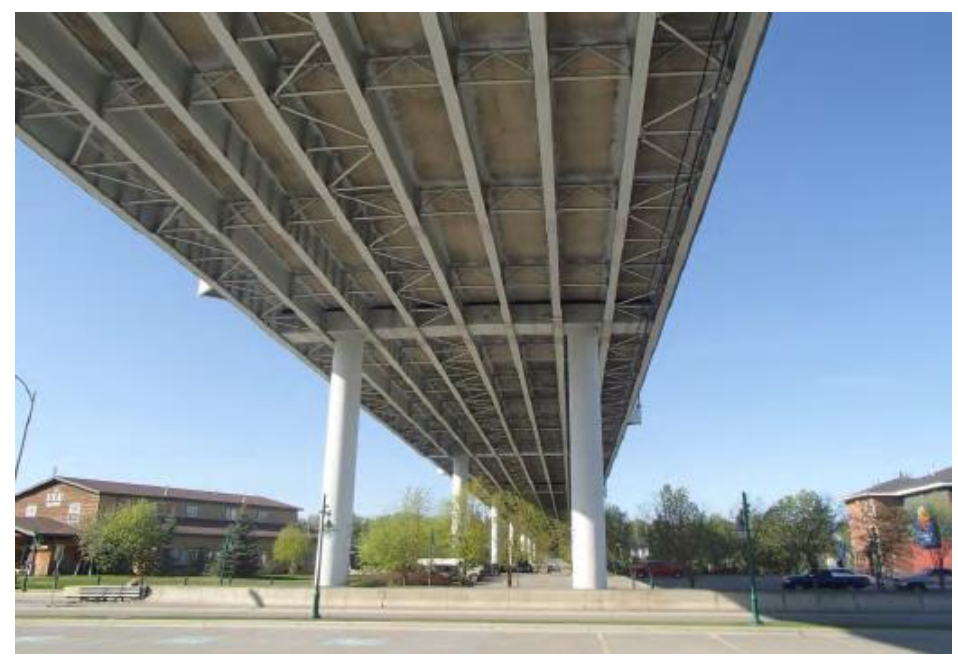

Figura 1: Puente Achorage Port Access

Fuente: (Calderón et al., 2018).

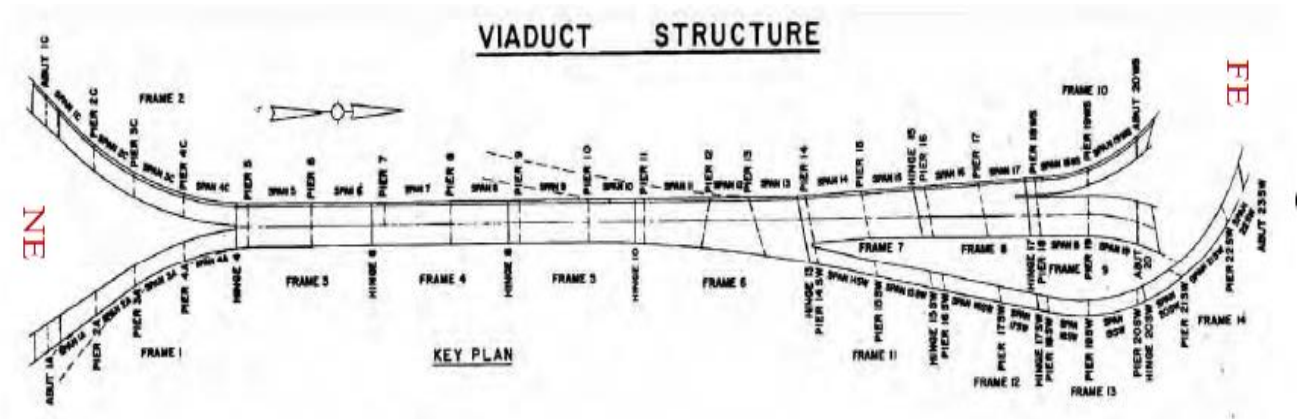

Figura 2: Vista en planta del Puente Achorage Port Access

Fuente: (Calderón et al., 2018). 
En un estudio previo (proyecto Anchorage Port Access, Universidad Estatal de Carolina del Norte) se detectó que el $61 \%$ de los "piers" presentaban cedencia de la viga de techo, antes que las columnas alcancen su momento plástico (Calderón et al., 2018).

En la Figura 3, se observan las formas de falla deseada y real. En la primera, hay daño moderado en columnas y no enviga, en la segunda, las columnas sufren daño grave y, peor aún, las vigas también se dañan. Nótese que la primera forma, deseada, sigue los lineamientos de diseño sísmico de la AASHTO (2007) para un puente tipo 1, los mismos que toman en cuenta la formación de rótulas plásticas en las columnas. En estos lineamientos, la subestructura debe ser dúctil y la superestructura debe permanecer esencialmente elástica.
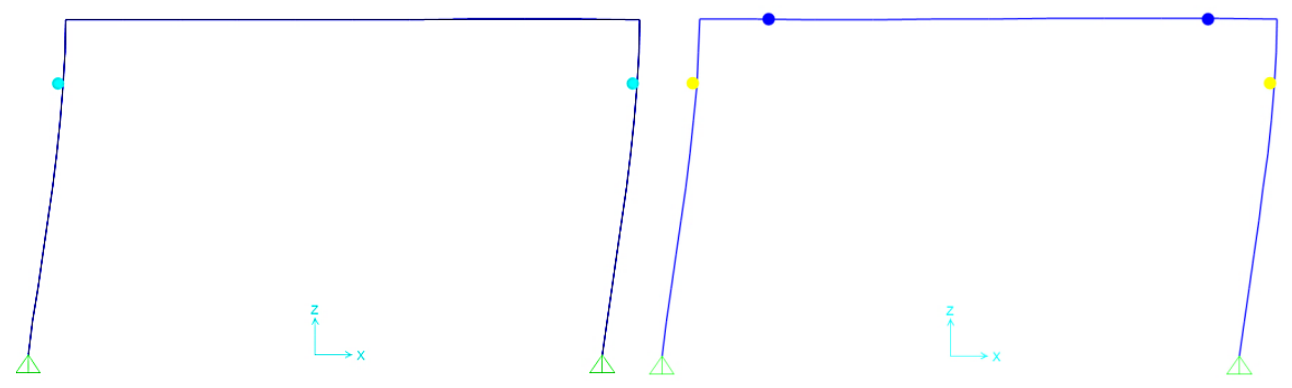

a) Falla ductil deseada

b) Modo real de falla

Figura 3: Esquema de modos de falla deseado y actual

Fuente: (Calderón et al., 2018).

En el modo real de falla, que representa el estado actual del $61 \%$ de los marcos, las rótulas plásticas también se están formando en las vigas de techo, causando que los factores de sobrerresistencia de la conexión viga-columna sean menores. Esto desencadena grandes deformaciones en la zona de las rótulas plásticas, evitando que los elementos estén protegidos por capacidad como lo menciona la AISC 341-10 (2010). Los factores de sobrerresistencia se refieren a la relación: momento de cedencia de la viga de techo $\left(M y_{C B}\right)$ dividido para momento de la viga de techo que iguala al de la formación de la rótula plástica en la columna $\left(M p_{C B}{ }^{c o l}\right)$.

La Tabla 1 muestra la denominación de los "piers", la relación diámetroespesor $(D / t)$ de la columna y el factor de sobrerresistencia. La relación $D / t$ determina la compacidad de las secciones estructurales y actualmente el límite mínimo para zonas sísmicas es de 35 para perfiles HSS compactos y 96 para no compactos, que corresponden a las relaciones $0.09 E / F_{y}$ y $0.044 E / F_{y}$, respectivamente. (AISC 341-10,2010). Además, se evidencia que la mayoría de geometrías incumplen con el valor mínimo de sobrerresistencia determinado en las especificaciones Caltrans (2016) y WSDOT M 23-50 (2018), respaldadas por la AASHTO (2007). Estas mencionan que, para que un elemento estructural esté protegido por capacidad, esta relación debe tener un valor mínimo de 1.25. Este valor puede ser 1 , si las condiciones de suelo son especialmente favorables.

Tabla 1: Datos de los diferentes "piers" del puente APA Fuente: (Calderón et al., 2018). 


\begin{tabular}{|c|c|c|}
\hline Pier & $\begin{array}{c}\text { Columna } \\
\text { D/ t }\end{array}$ & $\begin{array}{c}\text { Factor de } \\
\text { sobrerresistencia }\end{array}$ \\
\hline 2A & 96 & 2.18 \\
\hline $3 \mathrm{~A}$ & 96 & 1.81 \\
\hline 4A & 96 & 1.87 \\
\hline $2 \mathrm{C}$ & 96 & 1.64 \\
\hline 3C & 96 & 1.71 \\
\hline $4 \mathrm{C}$ & 96 & 1.53 \\
\hline 5 & 80 & 0.96 \\
\hline 6 & 80 & 0.96 \\
\hline 7 & 80 & 0.97 \\
\hline 8 & 80 & 0.97 \\
\hline 9 & 80 & 0.96 \\
\hline 10 & 80 & 0.96 \\
\hline 11 & 80 & 0.95 \\
\hline 12 & 69 & 0.80 \\
\hline 13 & 44 & 1.12 \\
\hline 14 & 80 & 1.15 \\
\hline 15 & 69 & 0.99 \\
\hline 16 & 44 & 0.83 \\
\hline 17 & 44 & 0.76 \\
\hline 18 & 96 & 0.81 \\
\hline 19 & 96 & 0.82 \\
\hline
\end{tabular}

El hecho de que el factor de sobrerresistencia es menor en muchos casos a 1.25, produce gran incertidumbre acerca de los efectos que puede llegar a desencadenar sobre el desempeño del puente. Por ejemplo, si las columnas alcanzan grandes deformaciones, se generarían fuertes esfuerzos que se concentrarían en las soldaduras de la conexión viga-columna, provocando que la viga no entre en fluencia y la soldadura falle (Goodnight, Kowalsky, \& Nau, 2013). En caso de que la soldadura esté perfectamente elaborada, la columna alcanzaría su momento plástico, pero la viga de techo fluiría antes (AISC 341-10,2010). Sin embargo, también debería considerarse que el proceso de suelda produce un cambio adicional en las propiedades del material.

Debido a las fuertes dudas sobre el comportamiento de la estructura, el proyecto APA va a desarrollar un ensayo sobre la conexión viga-columna del puente que caracterice su desempeño sísmico actual. Para ello, es necesario considerar un protocolo de carga que genere las condiciones críticas de comportamiento estructural. Sin embargo, debido a que la subestructura está construida enteramente de acero A36, se dificulta la elección de un protocolo de carga adecuado. No existe en los códigos actuales lineamientos para ensayar bajo cargas sísmicas puentes completamente hechos de acero (Shen, Yen, \& O'fallon, 2004).

En este artículo se presenta una investigación analítica computacional utilizando SeismoStruct para evaluar la capacidad de desplazamiento para un rango de bajos factores de sobrerresistencia. Para ello, se simularán diferentes tipos de historiales de carga para pruebas cuasi estáticas, con el propósito de 
establecer el más adecuado para el estudio minucioso del desempeño de los miembros del puente.

\section{METODOLOGÍA}

Tomando en cuenta que la experimentación debe ser un ensayo cíclico cuasi estático, porque debido a su baja velocidad facilita realizar las modificaciones necesarias para ajustarlo a circunstancias imprevistas (Shen et al.,2004), se modelarán varios marcos con diferentes secciones de vigas de techo a diferentes niveles de ductilidad. El propósito es obtener distintos valores del factor de sobrerresistencia $(\Omega)$.

Bruneau et al. (2016) y AISC 341-10 (2010) mencionan que la relación $\mathrm{My}_{\mathrm{CB}} / \mathrm{Mp}_{\mathrm{CB}}{ }^{\mathrm{col}}$ debe tener un valor mayor a 1.8 para zonas sísmicas. Por lo tanto, para simular las condiciones en las que la viga no está protegida por capacidad se va a variar $(\Omega)$ dentro del rango 0.9 a 1.8 .

Como se anotó anteriormente, en la actualidad no hay ningún código que provea de especificaciones para ensayar puentes de acero o sus componentes bajo ciclos de carga que representen los eventos sísmicos a los que está sujeto al ubicarse en una zona con alta peligrosidad sísmica. No obstante, varios autores como Stewart \& Val (1999), Goodnight et al. (2013), Fulmer et al. (2015) y Lanning et al. (2016), proponen algunas alternativas para ensayar este tipo de estructuras, basados en códigos vigentes como el ATC-24, FEMA-361 and AISC-341. Además, existe una propuesta que no está basada en los códigos planteada por Ge et al. (2013).

Los antes mencionados historiales de carga que se estudiarán como alternativas de aplicación se sintetizan en la Tabla 2. En ella se encuentra el enfoque principal de cada propuesta, el objetivo del ensayo, su forma de aplicación, el tipo de sismo al que es expuesto, el rango de desempeño, así como el protocolo de carga en sí.

Tabla 2: Tipos de protocolos de carga que serán estudiados

\begin{tabular}{|c|c|c|c|c|}
\hline Propuesta & FEMA 461 & AISC 341 & ATC-24 & $\begin{array}{l}\text { Ge et al. } \\
\text { (un ciclo) }\end{array}$ \\
\hline Enfoque & $\begin{array}{l}\text { Edificios y/o partes } \\
\text { dónde el daño se } \\
\text { predice mejor mediante } \\
\text { deformaciones } \\
\text { impuestas. }\end{array}$ & Edificios & $\begin{array}{ll}\text { Componentes } & \text { de } \\
\text { estructuras de acero } & \end{array}$ & $\begin{array}{l}\text { Estructuras } \\
\text { metálicas de } \\
\text { puentes }\end{array}$ \\
\hline Objetivo & $\begin{array}{l}\text { Ensayar especímenes } \\
\text { con un plan de } \\
\text { experimentación bien } \\
\text { definido cuyo protocolo } \\
\text { de carga permita } \\
\text { establecer una relación } \\
\text { entre los miembros en } \\
\text { diferentes estados de } \\
\text { daño con las demandas } \\
\text { asociadas. }\end{array}$ & $\begin{array}{ll}\text { Conexión de } & \text { momento } \\
\text { viga-columna } & \\
\text { Satisfacer } & \text { los } \\
\text { requerimientos } & \text { de } \\
\text { resistencia y ángulo de } \\
\text { deriva de piso. }\end{array}$ & $\begin{array}{l}\text { Prueba para un solo vano } \\
\text { Analizar el modo de falla } \\
\text { de la deterioración lenta } \\
\text { de la capacidad. }\end{array}$ & $\begin{array}{l}\text { Estudiar el } \\
\text { desempeño de la } \\
\text { fatiga extrema en } \\
\text { puentes de acero }\end{array}$ \\
\hline
\end{tabular}




\begin{tabular}{|c|c|c|c|c|}
\hline Sismo tipo & Ordinario & Ordinario & Ordinario & Ordinario \\
\hline $\begin{array}{l}\text { Rango de } \\
\text { prueba }\end{array}$ & $\begin{array}{l}\text { Elástico y/o } \\
\text { Plástico }\end{array}$ & $\begin{array}{l}\text { Elástico y/o } \\
\text { Plástico }\end{array}$ & $\begin{array}{l}\text { Elástico y/o } \\
\text { Plástico }\end{array}$ & $\begin{array}{l}\text { Elástico y/o } \\
\text { Plástico }\end{array}$ \\
\hline $\begin{array}{l}\text { Cómo } \\
\text { conducir el } \\
\text { ensayo }\end{array}$ & $\begin{array}{l}\text { Aplicación de ciclos } \\
\text { lentos de deformación. }\end{array}$ & $\begin{array}{l}\text { Controlando el ángulo } \\
\text { de deriva de piso }(\theta) \\
\text { impuesto para el ensayo } \\
\text { del espécimen. }\end{array}$ & 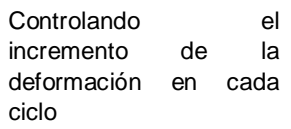 & $\begin{array}{l}\text { Controlando el } \\
\text { incremento de la } \\
\text { deformación en } \\
\text { cada ciclo }\end{array}$ \\
\hline $\begin{array}{l}\text { Protocolo de } \\
\text { carga }\end{array}$ & 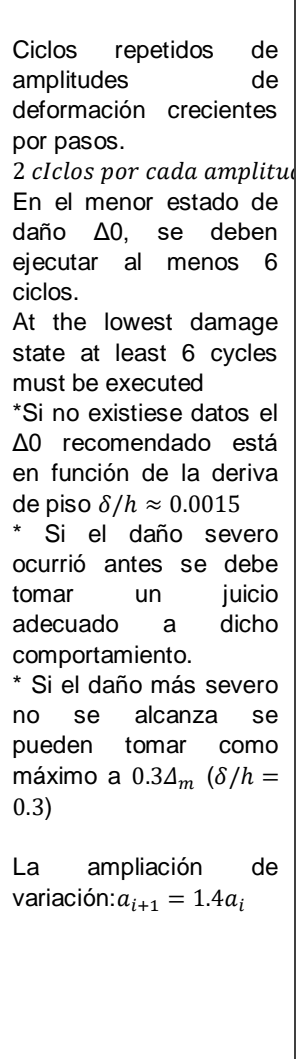 & 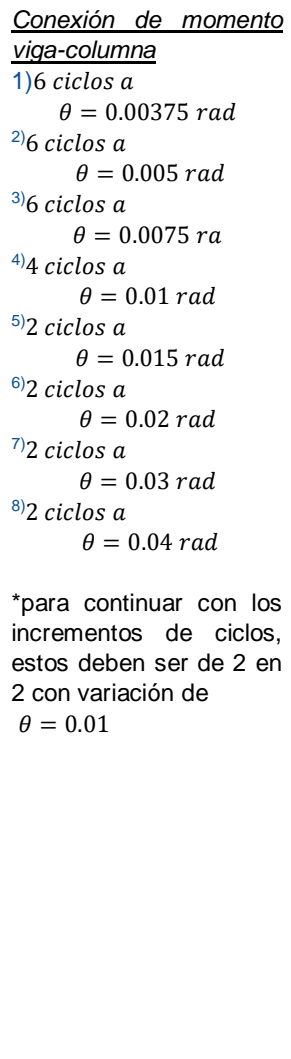 & $\begin{array}{l}\text { El número de ciclos } n_{o} \\
\text { con picos de } \\
\text { deformación } \quad \delta_{y} \\
\text { debería ser al menos } 6 \\
\left(n_{0}=\text { al menos } 6 \text { con: }\right. \\
\left.\delta_{0}<\delta_{y}\right) \\
{ }^{1)} n_{1}=\text { al menos } 3 \text { con } \\
\qquad \delta_{1}=\delta_{y} \\
{ }^{2} n_{2}=\text { al menos } 3 \text { con } \\
\delta_{2}=\delta_{y}+\Delta \\
{ }^{3} n_{3}=\text { al menos } 3 \text { con } \\
\delta_{3}=\delta_{y}+2 \Delta \\
{ }^{4} n_{4} \text { to } n_{n}=\text { al menos } 2 \\
\text { con: } \delta_{4}=\delta_{y}+3 \Delta \text { hasta } \\
\delta_{n}=\delta_{y}+m \Delta \\
\text { *a menos que un menor } \\
\text { número de ciclos se } \\
\text { pueda justificar. } \\
\text { *se podría aplicar mayor } \\
\text { número de ciclos si: } \\
\text {-El rango de daño } \\
\text { plástico acumulado > } \\
\text { máx. sismo de prueba } \\
\text { predicho para el ensayo. } \\
\text {-El deterioro ocurre } \\
\text { durante un paso de } \\
\text { carga y se considera } \\
\text { apropiado evaluar la } \\
\text { tasa de deterioro a } \\
\text { través de ciclos extras } \\
\text { de igual amplitud de } \\
\text { carga. }\end{array}$ & $\begin{array}{l}\text { Similar al } \begin{array}{l}\text { ATC- } \\
24,\end{array} \text { pero } \\
\text { aplicando solo un } \\
\text { ciclo. }\end{array}$ \\
\hline
\end{tabular}

Fuente: (FEMA-361,2010), (AISC-341,2010), (ATC-24,1992) y (Ge et al., 2013)

Una vez definidos los protocolos de carga a analizar se va a calcular la ductilidad a través de un análisis tipo pushover-estático en 2D. Los materiales se indican en la Tabla 3. Como se señaló previamente, el acero del cual está construido el puente es A36, pero las propiedades mecánicas reales de dicha estructura son disttintas.

Tabla 3: Propiedades mecánicas del acero Fuente: (Calderón et al., 2018).

\begin{tabular}{l|ll}
\hline Elemento & Columna & Viga de techo \\
\hline Módulo de elasticidad & $2.0 \mathrm{E}+06[\mathrm{MPa}]$ & $2.0 \mathrm{E}+06[\mathrm{MPa}]$ \\
Resistencia última & $517[\mathrm{MPa}]$ & $250[\mathrm{MPa}]$
\end{tabular}




\begin{tabular}{ll|ll}
$\begin{array}{l}\text { Deformación unitaria del } \\
\text { acero }\end{array}$ & 0.003 & 0.003 \\
\hline
\end{tabular}

Las secciones se muestran en la Figura 4, en dónde la columna está construida con un perfil circular tipo HSS y la viga de techo por una sección tipo W.
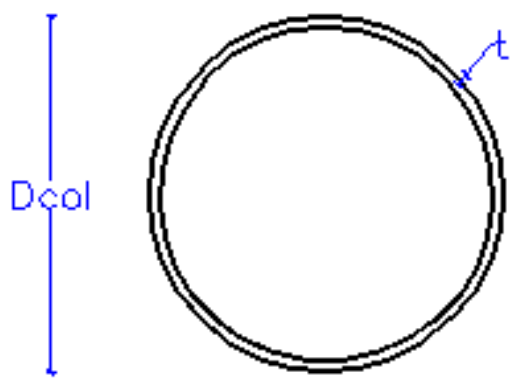

HSS-round

a) Configuración de la columna

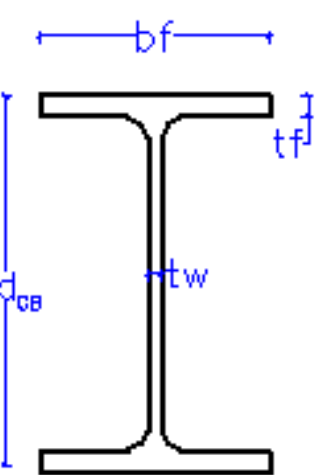

\section{Perfil W}

b) Configuración de la viga de techo

Figura 4: Tipos de perfil de los elementos del marco a analizar

Con el propósito de conocer el comportamiento del marco a de diferentes valores de $\Omega$ dentro del rango [0.9;1.8] y determinar su ductilidad, cuando este no esta protegido por capacidad, se optó por modelar 13 pórticos con diferentes secciones típicas de perfil W16, W14 y W12 para la viga de techo, mientras que, para la columna se escogió una única sección circular hueca HSS16X05. Dichas secciones se encuentran en la Tabla 4.

Para calcular los momentos que determinan el factor de sobrerrestistencia $\boldsymbol{\Omega}=\boldsymbol{M} \boldsymbol{y}_{\boldsymbol{C}} / \boldsymbol{M} \boldsymbol{p}_{C B}{ }_{\boldsymbol{B}}{ }^{o l}$, en las rótulas plásticas se empleó simple relación de triángulos como se observa en la Figura 5. Las dimensiones del marco son de $\mathrm{H}=3.50 \mathrm{~m}$ para las columnas y 3.65 de longitud para la viga de techo. Para el cálculo de la longitud de la rótula plástica se aplicó la ecuación (1) propuesta por ANSI/AISC 341-10 (2010), donde $h_{\text {hinge }}$ es la longitud de la rótula plástica, $d_{C B}$ es la altura efectiva de la viga de techo y $D_{c o l}$ es el diámetro de la columna. En la Tabla 4 se muestra además de los $\Omega$, la longitud de las rótulas plásticas.

$$
h_{\text {hinge }}=1 / 2\left(d_{C B}+D_{\text {col }}\right)
$$




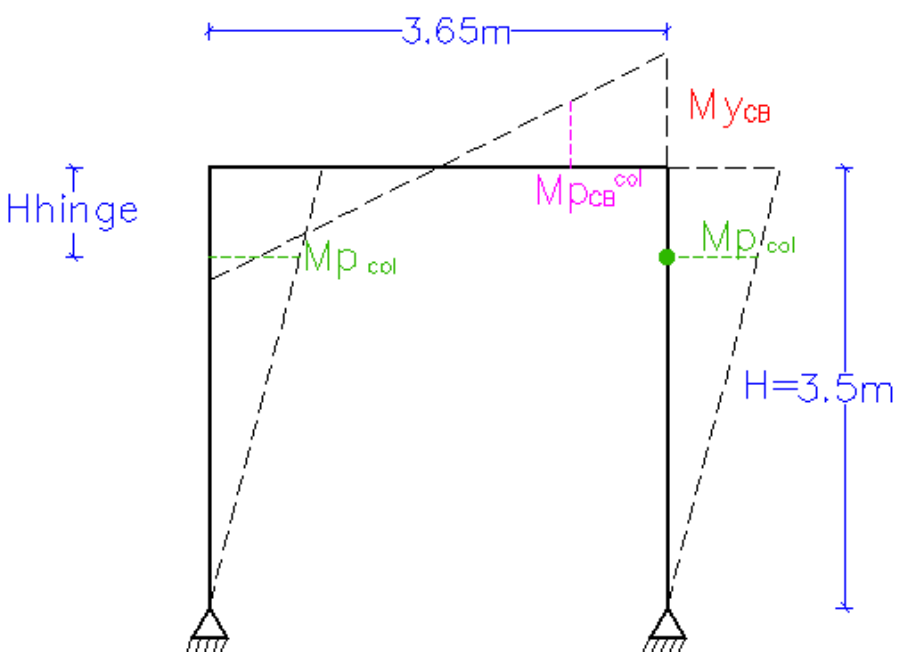

Figura 5: Diagrama de momento

Tabla 4: Diferentes factores $\Omega$ obtenidos a través del cálculo de los momentos

\begin{tabular}{|c|c|c|c|c|c|c|c|c|}
\hline COL. & \multicolumn{2}{|c|}{$\mathrm{CB}$} & $\begin{array}{c}H_{\text {hinge }} \\
{[\mathrm{cm}]}\end{array}$ & $\begin{array}{c}H-H_{\text {hing }} \\
{[\mathrm{cm}]}\end{array}$ & $\begin{array}{c}p_{\text {col }} \\
{[\text { ton } \cdot m]}\end{array}$ & $\begin{array}{l}M p_{C B}^{*} \\
{[\text { ton } \cdot m]}\end{array}$ & $\begin{array}{l}M y_{C B} \\
{[\text { ton } \cdot m]}\end{array}$ & $0.9<\frac{M y_{C B}}{M p_{C B}^{*}}<1.8$ \\
\hline \multirow{13}{*}{ HSS16x0.5 } & W12x136 & 13.4 & 37.34 & 313.18 & 83.79 & 80.30 & 77.31 & 0.96 \\
\hline & W12x152 & 13.7 & 37.72 & 312.80 & 83.79 & 80.30 & 86.87 & 1.08 \\
\hline & $W 12 \times 170$ & 14.0 & 38.10 & 312.42 & 83.79 & 80.30 & 97.67 & 1.22 \\
\hline & W12x190 & 14.4 & 38.61 & 311.91 & 83.79 & 80.30 & 109.31 & 1.36 \\
\hline & W12x230 & 15.1 & 39.50 & 311.02 & 83.79 & 80.30 & 133.42 & 1.66 \\
\hline & W12x252 & 15.4 & 39.88 & 310.64 & 83.79 & 80.30 & 146.72 & 1.83 \\
\hline & $W 14 \times 120$ & 14.5 & 38.74 & 311.79 & 83.79 & 80.30 & 78.97 & 0.98 \\
\hline & $W 14 \times 132$ & 14.7 & 38.99 & 311.53 & 83.79 & 80.30 & 86.87 & 1.08 \\
\hline & W14x145 & 14.8 & 39.12 & 311.40 & 83.79 & 80.30 & 96.43 & 1.20 \\
\hline & W14x159 & 15.0 & 39.37 & 311.15 & 83.79 & 80.30 & 105.57 & 1.31 \\
\hline & W14x193 & 15.5 & 40.01 & 310.52 & 83.79 & 80.30 & 128.85 & 1.60 \\
\hline & W14 1411 & 15.7 & 40.26 & 310.26 & 83.79 & 80.30 & 140.49 & 1.75 \\
\hline & $W 16 \times 100$ & 17.0 & 41.91 & 308.61 & 83.79 & 81.30 & 72.74 & 0.91 \\
\hline
\end{tabular}

Una vez calculados los factores $\Omega$, se procede con la obtención de las ductilidades $(\mu)$ mediante los resultados de la modelación realizada en SeismoStruct, en cual el criterio límite es la curvatura máxima $(\varnothing)$ experimentada por la viga de techo. Como se mostrará en el siguiente apartado, algunos marcos no alcanzaron este estado límite.

Para el cálculo de $\varnothing$ se aplicó la ecuación (2), donde $\varepsilon_{t}$ es la deformación unitaria del acero. Se utilizó esta fórmula ya que, asume que la distribución de las deformaciones permanece siempre lineal (Bruneu et al.,2016). El cálculo de este criterio límite para cada marco se muestra en la Tabla 5. Un marco tipo se presenta en la Figura 6: Marco tipo modelado en SeismoStruct, la viga de techo ha alcanzado su máxima curvatura 


$$
\emptyset=\varepsilon_{t} /\left(d_{C B} / 2\right)
$$

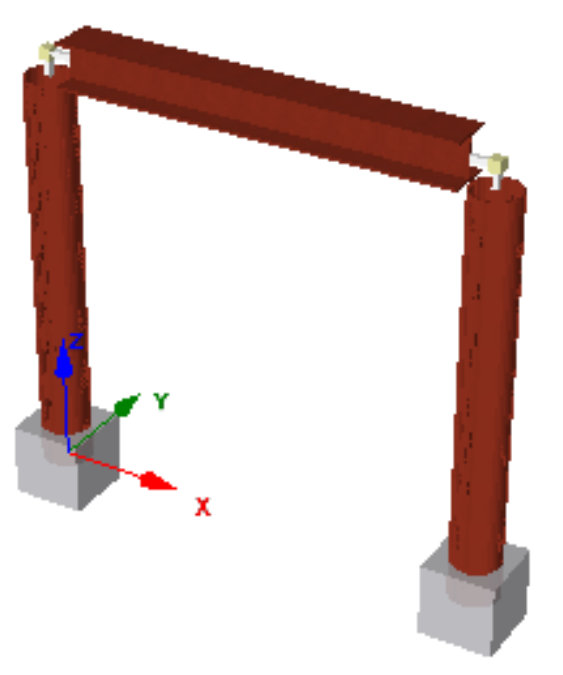

a) Marco antes de la aplicación del historial de cargas

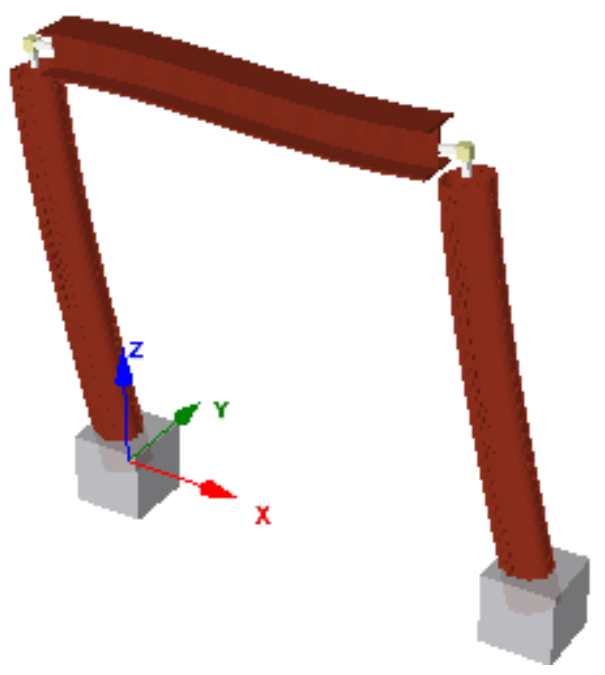

b) Marco después de completar el historial de cargas

Figura 6: Marco tipo modelado en SeismoStruct

\section{ANÁLISIS RESULTADOS}

Una vez que todos los marcos presentados en la Tabla 4 fueron modelados en el software SeismoStruct aplicando un análisis tipo pushover-estático, se obtuvieron los valores de desplazamiento de cedencia de la viga de techo $\left(\Delta y_{C B}\right)$ y el desplazamiento de la estructura $\left(\Delta_{y}\right)$, mismos valores que al aplicar la ecuación (3) se puede calcular los valores de ductilidad $\left(\mu_{C B}\right)$, dichos valores se muestran en la Tabla 5.

$$
\mu_{C B}=\Delta y_{C B} / \Delta_{y}
$$

\begin{tabular}{|c|c|c|c|c|c|c|}
\hline Section & $d_{C B}$ & Ratio & $\emptyset=\frac{\varepsilon_{t}}{d_{C B} / 2}$ & $\Delta y_{C B}$ & $\Delta_{y}$ & $\mu_{C B}=\frac{\Delta y_{C B}}{\Delta y}$ \\
\hline W12x136 & 13.4 & 0.96 & 0.00044776 & 2.47 & 2.47 & 1.00 \\
\hline W12x152 & 13.7 & 1.08 & 0.00043796 & 3.06 & 2.43 & 1.26 \\
\hline W12x 170 & 14.0 & 1.22 & 0.00042857 & 15.97 & 2.41 & 6.63 \\
\hline W12x190 & 14.4 & 1.36 & 0.00041667 & 24.50 & 2.36 & 10.40 \\
\hline$W 12 \times 230$ & 15.1 & 1.66 & 0.00039735 & 24.50 & 2.29 & 10.72 \\
\hline W $12 \times 252$ & 15.4 & 1.83 & 0.00038961 & 24.50 & 2.24 & 10.93 \\
\hline W14x120 & 14.5 & 0.98 & 0.00041379 & 2.46 & 2.46 & 1.00 \\
\hline W14x132 & 14.7 & 1.08 & 0.00040816 & 2.84 & 2.43 & 1.17 \\
\hline W14x 145 & 14.8 & 1.20 & 0.00040541 & 7.36 & 2.40 & 3.07 \\
\hline W14x159 & 15.0 & 1.31 & 0.00040000 & 23.47 & 2.37 & 9.91 \\
\hline W14x193 & 15.5 & 1.60 & 0.00038710 & 24.50 & 2.29 & 10.72 \\
\hline W14x211 & 15.7 & 1.75 & 0.00038217 & 24.50 & 2.25 & 10.91 \\
\hline W16x100 & 17.0 & 0.91 & 0.00035294 & 2.06 & 3.09 & 0.67 \\
\hline
\end{tabular}

Tabla 5: Valores de ductilidad para cada sección de las vigas de techo 
Es importante mencionar que las ductilidades obtenidas de la Tabla 5, hubiesen sido menores si se consideraba el efecto el pandeo de las columnas. Las secciones con $\mu_{C B}$ mayores son el resultado de grandes $\Delta y_{C B}$, esto se debe a que la viga de techo que conforman estos marcos es más fuerte, lo que implica que no alcanza el estado límite. Por otro lado, los valores menores de $\mu_{C B}$ se obtuvieron asumiendo que una vez que la viga de techo ha alcanzado $\varnothing$, el sistema se vuelve inestable. Los valores de $\mu_{C B}$ vs. los valores de $\Omega$ se muestran en la Figura 7 .

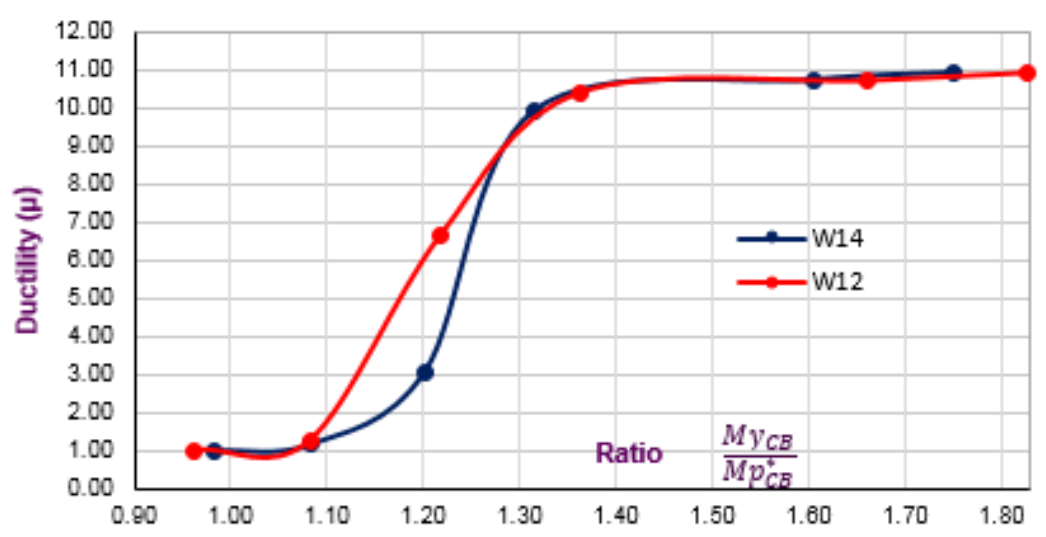

Figura 7: Valores de ductilidad vs $\Omega=M y_{C B} / M p_{C B}^{c o l}$

La tendencia de las curvas presentadas en la Figura 7, muestran como al tener mayores valores de $\Omega$, la ductilidad $\mu_{C B}$ se incrementa, además se observa como al aproximarse al límite de $\Omega=1.8$, los valores de ductilidad se vuelven constantes.

Finalmente, los valores de ductilidad obtenidos mediante el análisis tipo pushover-estático son utilizados para conocer la extensión de los historiales de carga, que se van a calcular en función del desplazamiento. Es así como los 13 marcos van a ser modelados empleando un análisis de historia en el tiempo para poder estudiar el comportamiento de estos ante cada uno de los protocolos de carga propuestos por el ATC-24, Ge et al. (2013) (un ciclo de carga), FEMA 361 y AISC 341.

Con el propósito de visualizar los distintos protocolos de carga, se graficó el historial del mismo para la sección de la viga de techo W14X132 cuyo $\Omega=1.08$, como lo muestran la Figura 8, Figura 9, Figura 10 y Figura 11. Adicionalmente, se graficó el punto en que ceden la columna (•column) y la viga de techo ( • cap beam) además se puede visualizar en que ciclo alcanza su estado límite (- - - limit state). 


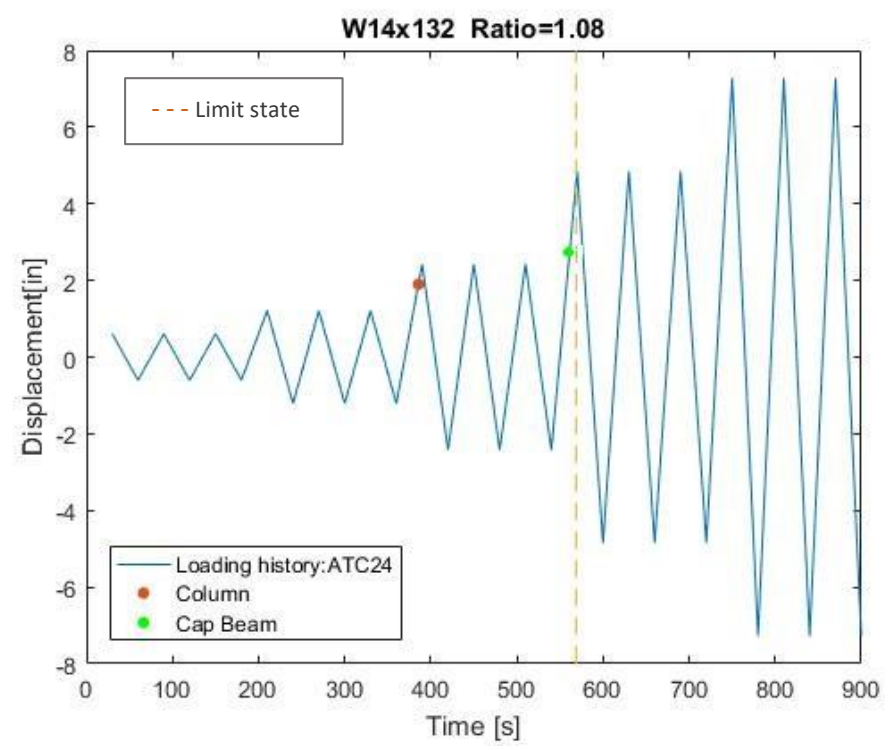

Figura 8: Protocolo de carga ATC-24

La Figura 8, muestra el historial de carga propuesto por el ATC en el cual el número total de ciclos es 13. Sin embargo, el estado límite se presenta al final del noveno ciclo. En cuanto a la cedencia de los elementos, la columna falla antes que la viga de techo en el sexto ciclo, mientras que la viga de techo lo hace en el noveno, previo a alcanzar el estado límite.

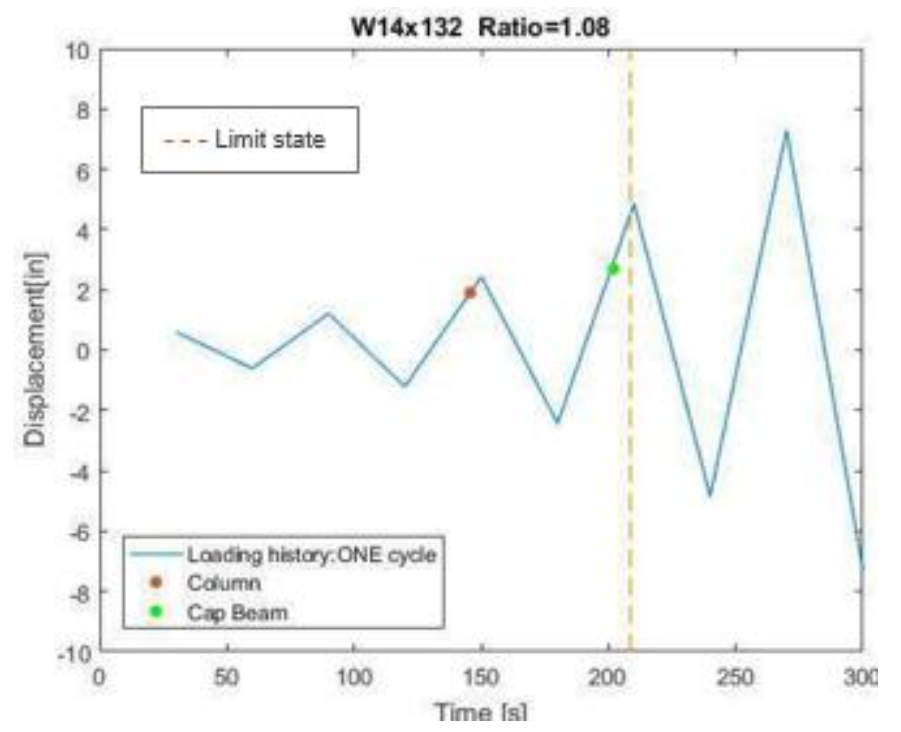

Figura 9: Protocolo de carga de un ciclo propuesto por Ge et al. (2013)

La Figura 9 representa un historial de cargas muy corto con apenas 4 y medio ciclos dónde la viga de techo y el estado límite se producen en el tercer 
ciclo, pero la cedencia de la viga se produce antes que alcance la curvatura máxima. Con respecto a la columna, esta falla en el ciclo número dos.

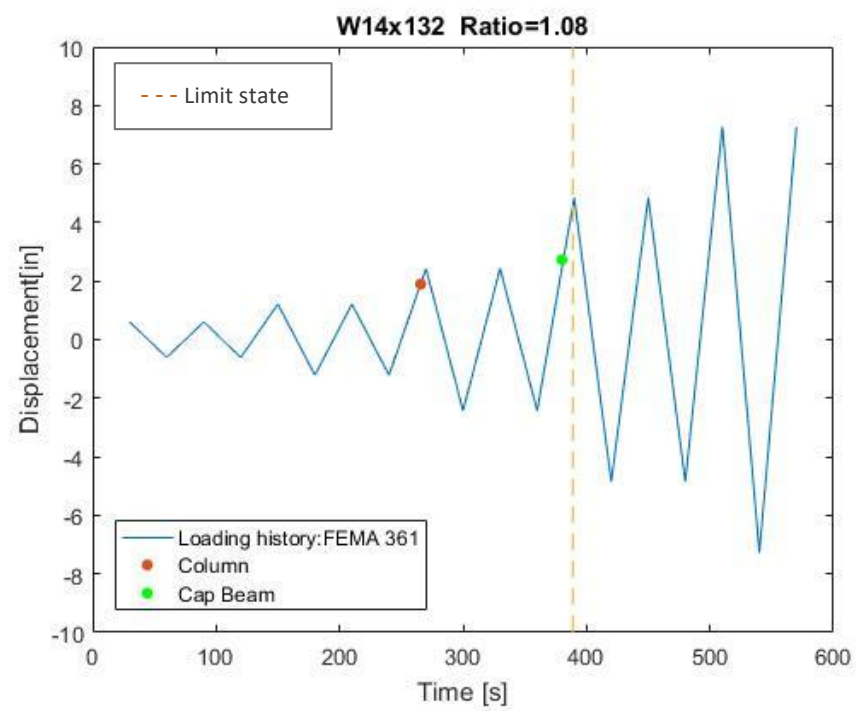

Figura 10: Protocolo de carga FEMA 361

Al igual que la Figura 8 y Figura 9, en la Figura 10 la viga de techo falla en el mismo ciclo que el estado límite, al final del ciclo 6 , mientras la columna alcanza el ciclo 4.

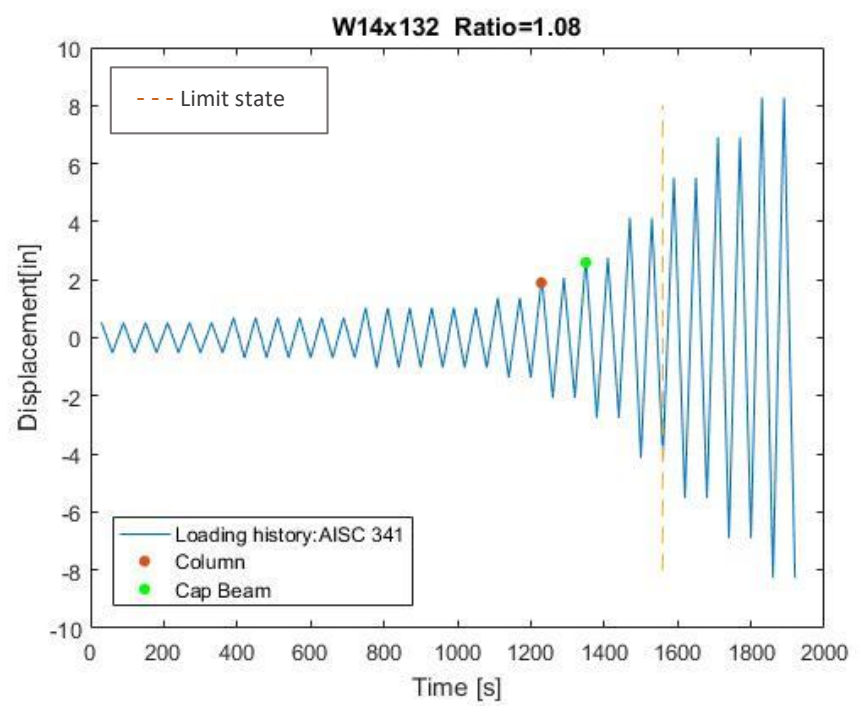

Figura 11: Protocolo de carga AISC 341 
Siguiendo el comportamiento de los protocolos anteriores, la columna falla antes que la viga de techo en el ciclo 20 y 21 y medio respectivamente. A diferencia de los historiales previos, su estado limite no ocurre igual que el de la viga de techo, sino en el ciclo número 25.

Para este tipo de sección de viga de techo, todos los marcos en cada uno de los protocolos de carga alcanzaron su estado límite. No obstante, existen casos en los cuales la viga de techo no logra experimentar su curvatura máxima. La respuesta de todos los marcos ante los cuatro historiales se sintetiza en las Tabla 6 y Tabla 7, donde la viga de techo está representada por la abreviación CB y la columna por COL.

Tabla 6: Resumen general de la respuesta del maro ante los historiales de carga aplicados en las diferentes secciones de vigas de techo

\begin{tabular}{|c|c|c|c|c|c|c|c|c|c|c|c|c|}
\hline \multirow{2}{*}{$\begin{array}{c}\text { Perfil de la } \\
\text { viga de } \\
\text { techo }\end{array}$} & \multicolumn{4}{|c|}{ Qué elemento falló primero } & \multicolumn{4}{|c|}{$\begin{array}{l}\text { Número de ciclos en el cual se alcanzó } \\
\text { el estado límite }\end{array}$} & \multicolumn{4}{|c|}{ Número total de ciclos } \\
\hline & ATC & 1 cycle & FEMA & AISC & ATC & 1 cycle & FEMA & AISC & ATC & 1 cycle & FEMA & AISC \\
\hline W12x136 & CB & CB & CB & CB & en 9 & en 3 & en 6 & en 28 & 11 & 3 & 9 & 17 \\
\hline W12x152 & CB & CB & CB & COL & en $121 / 2$ & en 9 & en 15 & en $281 / 2$ & 13 & 4 & 9 & 21 \\
\hline W12x170 & COL & CB & $\mathrm{COL}$ & COL & en 23 & en 11 & en $191 / 2$ & en 26 & 29 & 11 & 9 & 47 \\
\hline W12x190 & $\mathrm{COL}$ & $\mathrm{COL}$ & $\mathrm{COL}$ & $\mathrm{COL}$ & -- & -- & en $131 / 2$ & -- & 38 & 21 & 9 & 57 \\
\hline W12×230 & COL & $\mathrm{COL}$ & $\mathrm{COL}$ & $\mathrm{COL}$ & -- & -- & -- & -- & 38 & 13 & 9 & 57 \\
\hline W12×252 & COL & $\mathrm{COL}$ & $\mathrm{COL}$ & COL & -- & -- & -- & -- & 38 & 13 & 9 & 55 \\
\hline W14x120 & COL & $\mathrm{COL}$ & $\mathrm{COL}$ & COL & en 9 & en 3 & en 6 & en $231 / 2$ & 11 & 3 & 9 & 27 \\
\hline W14x132 & $\mathrm{COL}$ & $\mathrm{COL}$ & $\mathrm{COL}$ & $\mathrm{COL}$ & en 9 & en 3 & en 6 & en $251 / 2$ & 13 & 4 & 9 & 31 \\
\hline W14x145 & COL & $\mathrm{COL}$ & $\mathrm{COL}$ & $\mathrm{COL}$ & en 19 & en $71 / 2$ & en 12 & en 36 & 21 & 8 & 9 & 37 \\
\hline W14x159 & COL & $\mathrm{COL}$ & $\mathrm{COL}$ & COL & en 35 & -- & -- & -- & 38 & 12 & 9 & 55 \\
\hline W14x193 & $\mathrm{COL}$ & $\mathrm{COL}$ & $\mathrm{COL}$ & COL & -- & -- & -- & -- & 38 & 12 & 9 & 55 \\
\hline W14x211 & $\mathrm{COL}$ & $\mathrm{COL}$ & $\mathrm{COL}$ & $\mathrm{COL}$ & -- & -- & -- & -- & 38 & 12 & 9 & 55 \\
\hline W16x100 & $\mathrm{COL}$ & $\mathrm{COL}$ & $\mathrm{COL}$ & $\mathrm{COL}$ & en 6 & en 3 & en 4 & en 22 & 12 & 3 & 12 & 29 \\
\hline
\end{tabular}

Tabla 7:Resumen de la respuesta de cada elemento del marco, que fue sometido a diferentes protocolos de carga

\begin{tabular}{|c|c|c|c|c|c|c|c|c|c|c|c|c|}
\hline \multirow{3}{*}{$\begin{array}{l}\text { Perfil de } \\
\text { la viga } \\
\text { de techo }\end{array}$} & \multicolumn{8}{|c|}{ Número del ciclo en cual alcanzan la cedencia } & \multirow{2}{*}{\multicolumn{4}{|c|}{$\begin{array}{l}\text { Número de ciclos entre cada } \\
\text { cedencia }\end{array}$}} \\
\hline & \multicolumn{4}{|c|}{ Columna (COL) } & \multicolumn{4}{|c|}{ Viga de techo (CB) } & & & & \\
\hline & ATC & 1 ciclo & FEMA & AISC & ATC & 1 ciclo & FEMA & AISC & ATC & 1 ciclo & FEMA & AISC \\
\hline W12x136 & $31 / 2$ & $11 / 2$ & 4 & 20 & 3 & 2 & $31 / 2$ & 20 & $-1 / 2$ & $-1 / 2$ & $-1 / 2$ & 0 \\
\hline W12x152 & 6 & 2 & 4 & 20 & 6 & 2 & 4 & 21 & 0 & 0 & 0 & 1 \\
\hline W12x170 & 6 & 2 & 4 & 20 & $71 / 2$ & 2 & $41 / 2$ & $221 / 2$ & $11 / 2$ & 0 & $1 / 2$ & $21 / 2$ \\
\hline W12x190 & 6 & 2 & 4 & 20 & 21 & $51 / 2$ & & 36 & 15 & $31 / 2$ & 4 & 16 \\
\hline W12x230 & 6 & 2 & 4 & 20 & -- & -- & -- & -- & -- & -- & -- & -- \\
\hline W12x252 & 6 & 2 & 4 & 20 & -- & -- & -- & -- & -- & -- & -- & -- \\
\hline$W 14 \times 120$ & 6 & 2 & 4 & 20 & 6 & 2 & 4 & 22 & 0 & 0 & 0 & 2 \\
\hline W14x132 & 6 & 2 & 4 & 20 & 9 & 3 & 6 & $221 / 2$ & 3 & 1 & 2 & $21 / 2$ \\
\hline W14x145 & 6 & 2 & 4 & 20 & $101 / 2$ & 3 & $61 / 2$ & $281 / 2$ & $41 / 2$ & 1 & $21 / 2$ & $81 / 2$ \\
\hline W14x159 & 6 & 2 & 4 & 20 & $201 / 2$ & $81 / 2$ & -- & $421 / 2$ & $141 / 2$ & $61 / 2$ & -- & $221 / 2$ \\
\hline W14x193 & 6 & 2 & 4 & 20 & -- & -- & -- & -- & -- & -- & -- & -- \\
\hline W14x211 & 6 & 2 & 4 & 20 & -- & -- & -- & -- & -- & -- & -- & -- \\
\hline W16x100 & 6 & 2 & 4 & 20 & $61 / 2$ & $21 / 2$ & $41 / 2$ & 20 & $1 / 2$ & $1 / 2$ & $1 / 2$ & 0 \\
\hline
\end{tabular}


Como se visualiza en la Tabla 7 , los números de ciclos entre la cedencia de la columna y la viga, cuando ésta corresponde al perfil W12X136, son negativos. Esto indiica que la viga de techo cedió antes que las columnas, como se predijo en los estudios preliminares de Calderón et al. (2018). Esto implica que la viga es más débil de la columna. Por lo tanto, el marco de secciones W12X136 (viga de techo) y HSSS160X0.5 (columna) no siguen la filosofía de diseño de columnas débiles y vigas fuertes que caracteriza un diseño dúctil (Moustafa, 2014).

\section{CONCLUSIONES}

Como resultado del presente estudio sobre los tipos de protocolos de carga para puentes con subestructura elaborada completamente de acero sujeta a bajos valores en el factor de sobrerresistencia, se concluyó que:

- Las vigas de techo que ceden antes que las columnas no están diseñadas siguiendo la filosofía de diseño de columnas débiles y vigas fuerte, lo que podría justificarse ya que, en el tiempo en que se construyó el puente, los criterios de diseño sísmico para puentes aún no se desarrollaban.

- Cuando los factores de sobrerresistencia son cercanos a 1.8, el marco no alcanza su estado límite lo que quiere decir que sí está protegido por capacidad. De la misma manera sucede con los valores mayores a $\Omega=1.35$ (que es la mitad del rango [0.9;1.8]). Por lo tanto, este rango se podría reducir.

- A pesar de que se reduzca a la mitad, sigue estando por debajo de lo recomendado por los códigos de Caltrans (2007) y WSDOT (2018).

- Al comprar los historiales de carga que son casi similares, es decir, el ATC-24 de tres ciclos y el de Un ciclo de $\mathrm{Ge}$ et al. (2013), se podría optar por el primero, ya que en el ATC-24 el ensayo tiene mayor duración y experimenta una mayor cantidad de ciclos de carga.

- Siguiendo la misma condición para la elección de un protocolo de carga entre el AISC 341 y FEMA361, se optaría a simple vista por el que experimenta mayor cantidad de ciclos. Sin embargo, la mayoría de los ciclos experimentados en el AISC y FEMA se desarrollan en el rango elástico, por lo que el estudio del comportamiento actual del puente no entra en este rango.

- Finalmente, se optó por el historial de carga recomendado por el ATC-24 debido a que, experimenta mayor número de ciclos de carga en el rango plástico y la diferencia entre el número de ciclo en el que falla cada elemento es suficiente para que se analicen los posibles modos de falla que se pueden suscitar en la experimentación.

\section{TRABAJO FUTURO}

A fin de realizar un estudio más detallado, es necesario realizar un análisis que tome en cuenta el efecto del pandeo en las columnas del marco.

Realizar un estudio con modelos 3D de un vano esviajado para ver cómo le afectan las cargas sísmicas a un marco cuyo valor de $\Omega$ sea pequeño. 
Llevar a cabo un estudio analítico computacional acerca de cómo es el comportamiento del marco con valores bajos del factor de sobrerresistencia cuando la columna ya no es circular hueca, sino oblonga hueca, debido a que existen otros "piers" donde las columnas tienen este tipo de sección.

Desarrollar pruebas experimentales en la Universidad Estatal de Carolina del Norte utilizando los resultados de este estudio a fin de ensayar una conexión viga-columna, que presente un valor bajo de sobrerresistencia, a fin de, determinar el modo de falla que se producen.

\section{AGRADECIMIENTOS}

Los autores desean agradecer a la Universidad de las Fuerzas ArmadasESPE por apoyar esta investigación a través de una subvención, al igual que a la Universidad Estatal de Carolina del Norte donde se desarrolló la investigación. Un reconocimiento especial para el Dr. Mervyn Kowalsky y la Dra. Ana G. Haro, quienes participaron activamente en esta investigación como contactos técnicos, además de todo el personal y los estudiantes de posgrado del Constructed Facilities Lab (CFL) y al proyecto de Anchorage Port Access Bridge para permitirnos ser parte de él.

\section{REFERENCIAS}

1. Federal Emergency Management Agency (FEMA 461). (2007). Interim Testing Protocols for Determining the Seismic Performance Characteristics of Structural and Nonstructural Components.

2. Alaska Seismic Hazards Safety Commission (ASHSC). (2019, January 9). Earthquake Risk in Alaska. Retrieved from http://seismic.alaska.gov/earthquake_risk.html

3. American Institute of Steel Construction (AISC 341-10). (2010). Seismic Provisions for Structural Steel Buildings. Chicago, Illinois 60601-1802: AISC Committee on Specifications.

4. ANSI/AISC 341-10. (2010). AISC Seismic Provisions for Structural Steel Buildings. American Institute of Steel Construction.

5. Applied Technology Council (ATC-24). (1992). Guildelines for cyclic seismic testing of components of steel structures.

6. Bruneau, M., Uang, C. M., \& Whittaker, A. (2001). Ductile design of steel structures. New York: McGraw-Hill.

7. Calderón, V., Palma, A., Kowalsky, M., \& Nau, J. (2018). Achorage Port Access Bridge Proyect. Proyect developed in Constructed Facilities Lab (CFL)at NCSU as part of AlaskaDOT: Department of Civil, Construction and Environmental Engineering,. 
8. Fulmer, S., Kowalsky, M., \& Nau, J. (2015). Grouted shear stud connection for steel bridge substructures. Journal of Constructional Steel Research, 109, 72-86. doi:https://doi.org/10.1016/j.jcsr.2015.02.009

9. Ge, H., Kang, L., \& Tsumura, Y. (2013). Extremely Low-Cycle Fatigue Tests of Thick-Walled Cycle Fatigue Tests. Journal of Bridge Engineering, Vol. 18, Issue 9. doi:https://doi.org/10.1061/(ASCE)BE.1943-5592.0000429

10. Goodnight, J. C., Kowalsky, M. J., \& Nau, J. M. (2013). Effect of Load History on Performance Limit States of Circular Bridge Columns. Journal of Bridge Engineering, $\quad$ Volume $18 \quad$ Issue 12. doi:https://doi.org/10.1061/(ASCE)BE.1943-5592.0000495

11. Lanning, J., Benzoni, G., \& Uang, C.-M. (2016). Using Buckling-Restrained Braces on Long-Span Bridges. I:Full-Scale Testing and Design Implications. Journal of Bridge Engineering, Volume 21 Issue 5. doi:https://doi.org/10.1061/(ASCE)BE.1943-5592.0000781

12. Moustafa, M. A.-R. (2014). Structural Behavior of Bent Cap Beams in Asbuilt and Retrofitted Reinforced Concrete Box-Girder Bridges. Ph.D. dissertation, University of California, Berkeley.

13. Shen, J.-D., Yen, W., \& O'fallon, J. (2004). Handbook for seismic performance testing of bridge piers. Vancouver, B.C., Canada : 13th World Conference on Earthquake Engineering, Paper No. 1682.

14. Stewart, M. G., \& Val, D. V. (1999, July). Role of Load History in ReliabilityBased Decision Analysis of Aging Bridges. Journal of Structural

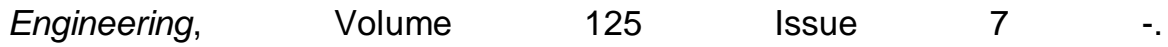
doi:https://doi.org/10.1061/(ASCE)0733-9445(1999)125:7(776) 